\title{
The control system of the grinding machine is designed for the rubber tree push-type gluing knife field
}

\author{
ZHANG Yuan ${ }^{1, a}$, DENG Yiguo ${ }^{1 *}, \mathrm{~b}$, WANG Yeqin ${ }^{1, \mathrm{c}}$, WEI Lijiao ${ }^{1, \mathrm{~d} a n d}$ Luo \\ Shiqiao $2,3,4, \mathrm{e}$
}

\begin{abstract}
${ }^{1}$ Tropical Agricultural Machinery Research Institute, Chinese Academy of Tropical Agricultural Sciences, Zhanjiang, China
\end{abstract}

${ }^{2}$ Rubber Research Institute, Chinese Academy of Tropical Agricultural Sciences, Danzhou, China

${ }^{3}$ The Center of CangLong Agricultural Technology Service for Zhanjiang City, Zhanjiang,China

${ }^{4}$ Agricultural Science and Technology Co., Ltd. of YIWU, Zhanjiang,China

azhang198414@126.com, bdyg7810@21cn.com, c441213852@qq.com, dweilijiao2008@163.com, e418727981@qq.com

\begin{abstract}
Keywords: Push.cut plastic knife.grinding machine.control system.design.
Abstract. Rubber tree push the cutter blade in the work of easy to glue, not wear and artificial tool grinding requires professional operation, and the workload is relatively large, longer, less efficient, the article for the above technology status quo. In this paper, the design idea, working principle and structural characteristics of the mechanical design are expounded, and the design model and implementation method of the control mechanism are introduced in detail. For the rubber tree Tapping tool grinding technology and supporting equipment optimization and innovation has a strong reference.
\end{abstract}

\section{Introduction}

Rubber Tree Cutter is an important tool for the production of natural rubber, there are two types of push and pull, the role is to cut the rubber tree bark regularly cut, so that the latex along the cut into the designated vessel, To facilitate the follow-up collection. At present, the tapping workers are widely used in the most of the cutting jacquard is a push cutter, is also the most widely used in a class of plastic knife. The plastic knife from the edge, the blade and the handle of the three main components, characterized by the edge of the general treatment with the processing of carbon steel tool, the blade is generally made of ordinary steel forging, handle with a touch of wood material, The overall cost is low, the cost can be easily rubber growers and tapping workers accepted. Of course, the kind of cutting plastic knife still exists a lot of deficiencies, such as: due to uneven quality of the material caused by the blade easy to glue, not wear, not sharp enough, easy to blunt and so on; grinding depends on the artificial grinding stone to complete, Large amount of work, long time, tapping efficiency is low, a lot of waste of manpower and material resources, and the use and maintenance of plastic gluing both need experienced master, professional requirements higher, hinder the sustainable development of the rubber industry.

In the history of the development of cutting plastic knife, in order to further improve the efficiency, to solve the problem of tool wear, after a long period of accumulation, with greater improvement and optimization, the market appeared a variety of improved cutting plastic knife ${ }^{[1-2]}$. One of the most common gluing knives, the main body including the steel body and installed in the tail of the knife handle. It is characterized by the use of good steel (usually felling on the process), and the back of the blade is made of ordinary steel or in the end of ordinary steel cutter body with wear-resistant steel , The advantage is to save the amount of high quality steel, knife blade is more sharp, not blunt, help to improve work efficiency and improve productivity, compared to all wear-resistant steel cutting tools, the cost is low, and no difference in the use and maintenance.

In foreign countries, rubber tree gluing knife technology and the status quo is similar, but also in the work efficiency and wear resistance to make a fuss, mainly in the emergence of a replacement blade cutting knife, in order to avoid every day sharpening to the plastic workers The burden of time, 
but after the time test, the improved cutting tool positioning accuracy is poor, unreliable operation, but also a large area and a long time to promote.

Through the comparative study of domestic and foreign cutting jelly knives can be found, pushing the cutting knife is still firmly maintained the mainstream status, but the use of the process still exists, specifically in: tapping tool is only applicable to professional tapping workers, Grinding is also the need for experienced professionals to spend more time to complete. Based on this, the problem to be solved in this study is how to quickly and accurately puncture the cutting blade cutting blade, making the relevant technical requirements have declined, so that more people can wear a knife, thereby improving the work efficiency of plastic workers.

\section{Machine design requirements and working principle}

Design requirements. The whole machine is designed to solve the problem of push-type gluing knives need to be polished by professionals and take a long time as the fundamental task, taking into account the following actual processing requirements:

a. The performance of the implementing agency shall meet the requirements of grinding of T10 and above applicable materials not less than those specified in GB / T 12981986 and shall meet the requirements of machining accuracy;

b. The control mechanism should be able to significantly improve the efficiency of the work, that is, one can complete the rapid clamping and multi-process work to solve the cost and efficiency of the two indicators;

c. The end of the actuator can be flexible to achieve the three degrees of freedom required for grinding the above needs.

Working principle. Taking into account the need to polish the cutting knife edge for the complex symmetrical space surface, in order to quickly grinding, need to be specially modified by the special molding wheel made of the edge of the molding grinding, while controlling the cutting knife blade before the knife The angle between the angle and the forming wheel, and the relative movement speed of the cutter and the forming wheel. So the process is determined as: start grinding machine $\rightarrow$ forming wheel repair type (not often type) $\rightarrow$ to be grinding blade grinding knife in a special fixture $\rightarrow$ adjust the cutting blade grinding angle $\rightarrow$ adjust the blade and grinding wheel grinding position $\rightarrow$ repeated manipulation Knife mechanism to achieve the grinding wheel edge of the cutting edge of the knife $\rightarrow$ stop unloading knife.

Machine design with reference to the principle of machine technology and structure, with a small desktop grinder, the first to wear abrasive knife to the end of a certain angle grinding, and then through the precise repair molding wheel (dedicated), multi-angle adjustment function dedicated fixture, feed The knife and other institutions to achieve the rubber tree cutter in this special machine tool on the fast clamping, grinding angle adjustment, feed and cutting edge fast precision grinding, while the completion of the two cutting knife sharpening. The whole process of cooling water circulation system for the entire grinding process of chip removal and cooling, to ensure the quality of sharpening. Specific working principle shown in Figure 1:

Figure 1 Schematic diagram of a sharpening machine

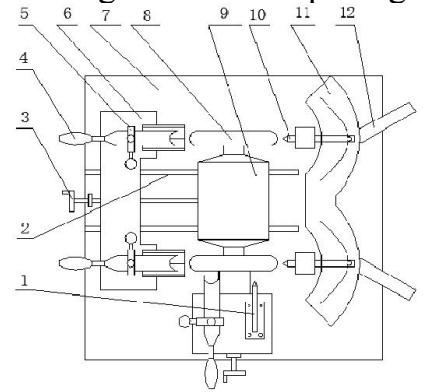

1 diamond pen, 2 linear guide, 3 feed wheel, 4 cutter, 5 special fixture, 6 grinding knife table, 7 base, 8 forming wheel, 9 double head grinder, 10 diamond pen, Positioning handle 
From the Figure 1 know, work, start the grinder, control the feed wheel so that the grinding wheel to the right to move to repair the location, and then lock, adjust the positioning handle after the grinding wheel to accurately repair ${ }^{[3]}$. After repairing the blade to be sharpened to the grinding position, with a special fixture clamping, the whole angle, positioning, control the knife table angle, and then control the feed wheel to gradually move the grinding wheel, And then take control of the knife blade began to sharpen the knife-edge, and finally remove the underwear with sandpaper repair small knife to the burr, cut plastic knife grinding work is completed.

Structural features. A. For the needs of the application, the whole structure is small, simple, low cost.B. Control system micro-operation feed, versatility, reduce the operator labor intensity, operation light and fast.C. Cutting blade grinding grinding folder is simple, fast, and can simultaneously sharpen two knives, a working time is less than 3 minutes, high efficiency.D. The use of diamond pen dressing wheel, relying on the mold positioning, to ensure that the grinding wheel grinding profile accurate, the blade grinding tool edge exactly the same, grinding blade quality is not affected by human factors.E. Workbench guide structure and feed, with linear guide and screw nut mechanism, reliable, light operation, high precision, to ensure the quality of grinding..

\section{Execute control system design}

Track realization. According to the Chinese Ministry of Agriculture released in July 2006 the standard: NY / 267-2006. At present, there are three kinds of knife-edge cutting knife knife knife, respectively: large round knife blade, small round knife blade.

Figure 2 by the track map

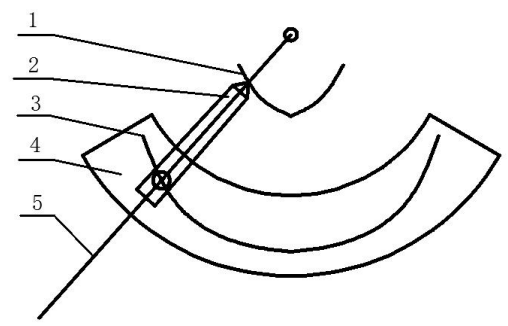

1 blade shape, 2 diamond pen, 3 by the mold track, 4, by the mold, 5 handle

Three kinds of knife-edge round mouth is more difficult to sharpen, round angle of $80 \pm 2$ degrees, half-angle of about 40 degrees, and the molding wheel need to use diamond pen repair, diamond pen angle is generally 60 degrees, half-angle 30 degrees The In order not to interfere with the grinding angle, while improving the accuracy and ease of use, the use of rotation plus linear movement to form the path of the knife (shown in Figure 2). It can be seen for different knife-edge cutting knife need a different rely on the mold, so the design series easy to replace the mold is to improve the efficiency and applicability of the cutter blade grinding the necessary conditions.

Feed control. In order to save the guide rail and improve the feed accuracy, the three-dimensional movement in the process of grinding and grinding is controlled by step linear displacement hydraulic control ${ }^{[4-5]}$. The grinding of the end face is done by the table feed.Refer to section 3 for simulation.

Figure 3. Simulation schematic

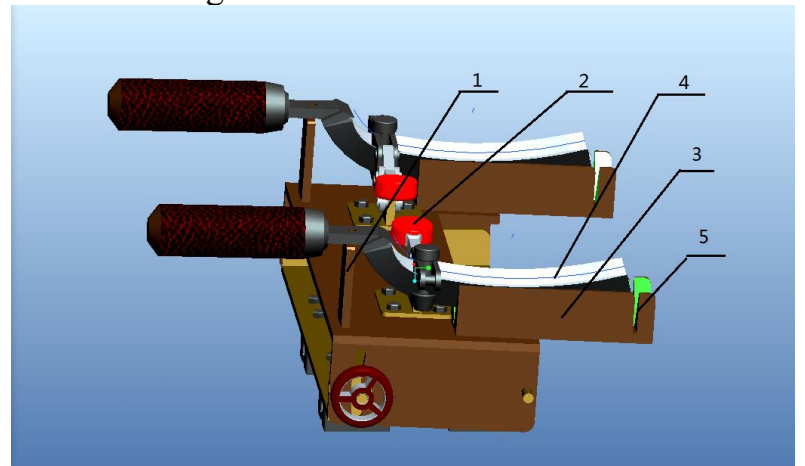

1 support plate, 2 quick fixture, 3 positioning block, 4 cut plastic knife, 5 positioning mouth 
When working, the linear control of the grinding wheel displacement. When the grinding wheel to the right and the grinding pen can be used to repair the type of feed, left when you can adjust the position of the grinding machine and cutting knife for cutting knife blade grinding mill.

The precision of the three-dimensional linear feed control is directly affected by the stepper motor. The output signal is controlled by the digital displacement sensor Feedback to the microcontroller controller with a given mechanical displacement comparison, the PID control after the control stepper motor correction hydraulic cylinder displacement output.

in the control system to the valve angle as the input signal to the hydraulic energy as the driving force, so that the hydraulic cylinder piston displacement according to the input signal changes in the $\mathrm{law}^{[6]}$. The force balance equation of the hydraulic cylinder is:

$$
m \frac{d^{2} y}{d t^{2}}=A p_{l}-B \frac{d y}{d t}-F-k \cdot y
$$

Remarks: $\mathrm{P}$ is the mechanical displacement; $\mathrm{A}$ is the effective working area of the hydraulic cylinder; $\mathrm{Pl}$ is the load pressure drop $\mathrm{Pl}=\mathrm{p} 1-\mathrm{p} 2$; $\mathrm{B}$ is the viscous damping coefficient; $\mathrm{F}$ is the external load; $\mathrm{k}$ is the spring stiffness of the load; $m$ is the moving part the quality.

According to the continuity equation, the flow balance equation of the left and right cavities can be obtained:

$$
q_{l}=A \frac{d y}{d t}+k_{1} \cdot p_{l}+\frac{V}{4 K} \cdot \frac{d p_{L}}{d t}=C_{d} \omega\left(x_{s}-x_{0}\right) \frac{p_{p}-p_{L}}{\rho}
$$

Remarks: Q1 is the flow rate of the input hydraulic cylinder; $\mathrm{k} 1$ is the total leakage coefficient of the hydraulic cylinder; $\mathrm{V}$ is the total volume of the two chambers of the hydraulic cylinder; $\mathrm{K}$ is the volume elastic modulus of the oil; $\mathrm{x} 0$ is the amount ; $\mathrm{Xs}$ is the amount of rotation (tangential) of the valve, proportional to the stepping motor angle; $\mathrm{pp}$ is the pump supply pressure; $\rho$ is the density of the oil; $\mathrm{Cd}$ is the flow coefficient of the valve; $\omega$ is the area of the oil hole.

After finishing, the transfer function of the factors such as load inertia, damping, stiffness and oil elasticity, hydraulic cylinder leakage and other factors can be considered. If the factors such as damping, spring rigidity and leakage are neglected, ) $=0$, the simplified transfer function from the spool rotation amount $\mathrm{xs}$ to the displacement $\mathrm{Y}$ of the piston output is obtained as follows:

$$
\frac{Y(s)}{x_{s}(s)}=\frac{K_{q}}{S \frac{V m S^{2}}{4 K A}+\frac{m K_{c} S}{A}+A}
$$

The transfer function of the entire displacement control system is shown in Figure 4.

Figure 4 Transfer function block diagram

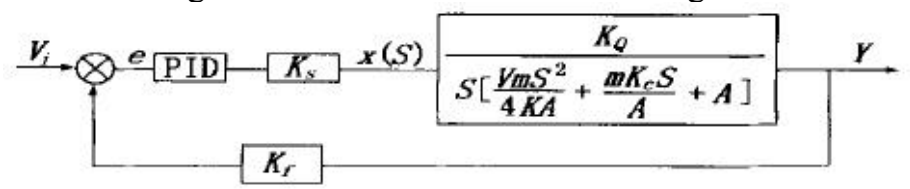

Remarks: $\mathrm{Kf}$ is the amplification factor of the digital displacement sensor, Ks is the magnification coefficient of the stepping motor output from the PID output to the switching valve, and PID is the control mode of the step number of the stepping motor.

Clamping mechanism. The local control actuator and the clamping mechanism have feeds such as leadscrews and sliders, and are equipped with a lock function. In order to prevent the vibration from stabilizing the positioning after feeding, the locking function is additionally increased.

Screw nut matching, through the connection flange to connect the grinding wheel base to drive the grinding machine feed, fast fixture to consider the use of the reliability and convenience and size of the non-interference, the choice of Taiwan GOOD HAND brand GH-22100 fast fixture.

\section{Conclusions}

By drawing on the technical structure and principle of the machine tool, combined with the actual characteristics of the cutter itself. This paper designs a kind of automatic cutting tool, which adopts the stepper motor to control the displacement output of the hydraulic cylinder. The output signal is 
fed back to the single-chip controller by the digital displacement sensor. Compared with the given mechanical displacement, the control output of the hydraulic cylinder is controlled by PID control, which can improve the control precision and stability remarkably. The combination of screw and slider mechanism can finish the automatic grinding of the cutter Of the action requirements for the cutting tool automatic grinding technology and supporting equipment optimization and innovation has a strong reference.

\section{Acknowledgements}

This work was financially supported by the Central Public-interest Scientific Institution Basal Research Fund for Chinese Academy of Tropical Agricultural Sciences (No.1630022015018\&1630132017003) and Collaborative innovation and platform environment construction special of Guangdong Province (2017A040406003).

I would like to thank the members of the research group for their selfless help, especially the communication author Deng Yiguo, associate professor and the Guangdong Ocean University School of Engineering to give the strong support.

\section{References}

[1] ZHANG Jianmin. Design of Mechatronic System[M].Beijing Polytechnic University Press.2000,2.

[2] CHEN Huiming. The Study on Step Linear Output Control[J].Journal of Nanjing Forest ry University (Natural Sciences Edition).2003(1):30-32.

[3] CHEN Ming. Mechanical Manufacturing Technology[M].Machinery Industry Press.2005,1.

[4] ZHANG Jin,OU Zhongqing,CHEN Xudong. The Study on Step Linear Output Control[J].Journal of Nanjing Forest ry University (Natural Sciences Edition).2003(1):30-32..

[5] NY/267-2006,Push the knife[S].

[6] LI Zhuo. Linear System Control Theory and Application in the Case of Limited Rate of Drives[D].Hei Longjiang.Harbin Institute of Technology.2015. 